\title{
Possibility of new shielding device for upper gastrointestinal endoscopy
}

\section{(c) (i)}

Authors

Daisuke Kikuchi' ${ }^{1}$, Daiki Ariyoshi ${ }^{2}$, Yugo Suzuki ${ }^{1}$, Yorinari Ochiai ${ }^{1}$, Hiroyuki Odagiri ${ }^{1}$, Junnosuke Hayasaka ${ }^{1}$, Masami Tanaka', Tetsuya Morishima², Keita Kimura², Hiroshi Ezawa ${ }^{3}$, Risa Iwamoto ${ }^{2}$, Yoshinori Matsuwaki ${ }^{4}$, Shu Hoteya ${ }^{1}$

Institutions

1 Department of Gastroenterology, Toranomon Hospital, Tokyo Japan

2 Olympus Medical Systems Corporation, Tokyo, Japan

3 Olympus Corporation, Tokyo, Japan

4 Matsuwaki Clinic Shinagawa, Tokyo, Japan

submitted 23.2.2021

accepted after revision 17.5.2021

\section{Bibliography}

Endosc Int Open 2021; 09: E1536-E1541

DOI 10.1055/a-1523-8959

ISSN 2364-3722

(C) 2021. The Author(s).

This is an open access article published by Thieme under the terms of the Creative Commons Attribution License, permitting unrestricted use, distribution, and reproduction so long as the original work is properly cited. (https://

creativecommons.org/licenses/by/4.0/)

Georg Thieme Verlag KG, Rüdigerstraße 14,

70469 Stuttgart, Germany

Corresponding author

Daisuke Kikuchi, Department of Gastroenterology,

Toranomon Hospital, 2-2-2 Toranomon, Minato-ku, Tokyo, Japan

Fax: +81-3-3582-7068

dkiku1230@gmail.com

\section{ABSTRACT}

Background and study aims Infection control is essential when performing endoscopic procedures, especially during the COVID-19 pandemic. Therefore, we have developed a new shielding device called STEP for infection control in upper gastrointestinal endoscopy.

Patients and methods STEP consists of a mask worn by the patient and a drape that is connected to the mask and covers the endoscope. A suction tube attached to the mask prevents aerosols from spreading. The endoscopist operates the endoscope through the drape. Three endoscopists performed a total of 18 examinations using an upper endoscopy training model with and without STEP. Endoscopic images were evaluated by three other endoscopists, using a visual analog scale. We also simulated contact, droplet, and aerosol infection and evaluated the utility of STEP. Results All examinations were conducted without a problem. Mean procedure time was $126.3 \pm 11.6$ seconds with STEP and $122.3 \pm 10.0$ seconds without STEP. The mean visual analog score was $90.7 \pm 10.1$ with STEP and $90.4 \pm 10.0$ without STEP. In the contact model, adherence of simulated contaminants was $4.9 \pm 1.4 \%$ without STEP and $0 \%$ with STEP. In the droplet model, the number of simulated contaminants attached to the paper was $338273 \pm 90735$ pixels without STEP and 0 with STEP. In the aerosol model, the total number of particles was $346837 \pm 9485$ without STEP and was significantly reduced to $222 \pm 174$ with STEP.

Conclusions No effect on examination time or endoscopic image quality was observed when using STEP in upper gastrointestinal endoscopy. Using STEP reduced the diffusion of simulated contaminants in all three infection models.

\section{Introduction}

Many cases of infection and death have been reported due to the coronavirus disease 2019 (COVID-19) pandemic. As of January 2021, more than 85 million cases of infection have been confirmed and the number of deaths has surpassed 1.8 million worldwide.

COVID-19 can develop in individuals infected by severe acute respiratory syndrome coronavirus 2 (SARS-CoV2), which is an RNA virus that belongs to the broader family of coronaviruses. The viruses that caused severe acute respiratory syndrome (SARS) and Middle East Respiratory Syndrome (MERS) were also coronaviruses [1]. SARS-CoV2 is thought to be transmitted mainly by contact infection and droplet infection, but the precise mechanisms are unknown. Recent reports have suggested the possibility of aerosol infection, and thus, more careful infection control measures are considered necessary [2-4]. Wearing masks and disinfecting the hands are effective for pre- 
venting infection, and medical institutions are also implementing various infection control measures. There is currently no established treatment, but the effectiveness of various antiviral drugs has been reported $[5,6]$. Vaccine development is being carried out all over the world, and further development is expected in the future [7-12].

Endoscopic procedures carry a risk of infection because there is a risk of exposure to the patient's respiratory secretions and digestive juices. For this reason, many academic societies have proposed infection control measures for endoscopic procedures [13-17]. When performing endoscopy, it is necessary for endoscopists to wear adequate personal protective equipment (PPE), disinfect their hands, and clean the endoscopy room. In addition to these steps, new steps for infection control that shield patients and prevent the spread of contaminants are considered necessary.

Many cities around the world have been placed on lockdown to slow the spread of the COVID-19 pandemic, which has had serious economic consequences. The pandemic has also affected endoscopic practice, making it difficult to perform conventional endoscopic examinations $[18,19]$. In response, we are developing the new "Shielding Device for Endoscopic Procedures" as part of the Save the Endoscopy Project (STEP by STEP). Here, we report the current status of the project and report a preliminary evaluation of the feasibility and utility of STEP.

\section{Patients and methods}

\section{Device description}

We are developing STEP in collaboration with Olympus Medical Systems Co., Ltd. and an otolaryngologist. STEP consists of a mask ( Fig. 1a) worn by the patient and a tubular drape ( $\mathbf{F i g . 1 b}$ ) that covers the endoscope. On the side of the mask, there is a small hole for air inflow and a suction port for suction of aerosols generated by the patient. The drape connects to a hole at the tip of the mask and the endoscope is inserted into the drape. There is also a valve-like structure in the hole that prevents droplets from escaping when the patient coughs.

\section{How to use}

The use of STEP is shown in > Fig. 1c, > Fig. 1d, > Fig. 1e. First, the endoscope is inserted into the tubular drape, which is then taped to the base of the endoscope. After pharyngeal anesthesia, the mask is placed on the patient's face and the patient is positioned in the left lateral position as usual. The suction tube is attached to the suction port. Next, the tip of the tubular drape is connected to the hole in the mask. The endoscopist operates the endoscope through the vinyl tubular drape. Because the endoscope is located inside the tubular drape and mask, the endoscopist does not come into direct contact with the patient's saliva or digestive juices. When the endoscopic procedure is completed, the endoscope is stored inside the tubular drape and the tubular drape is detached from the mask.

Given that the hole in the mask has a valve-like structure, it is difficult for droplets inside the mask to escape. The contamina-
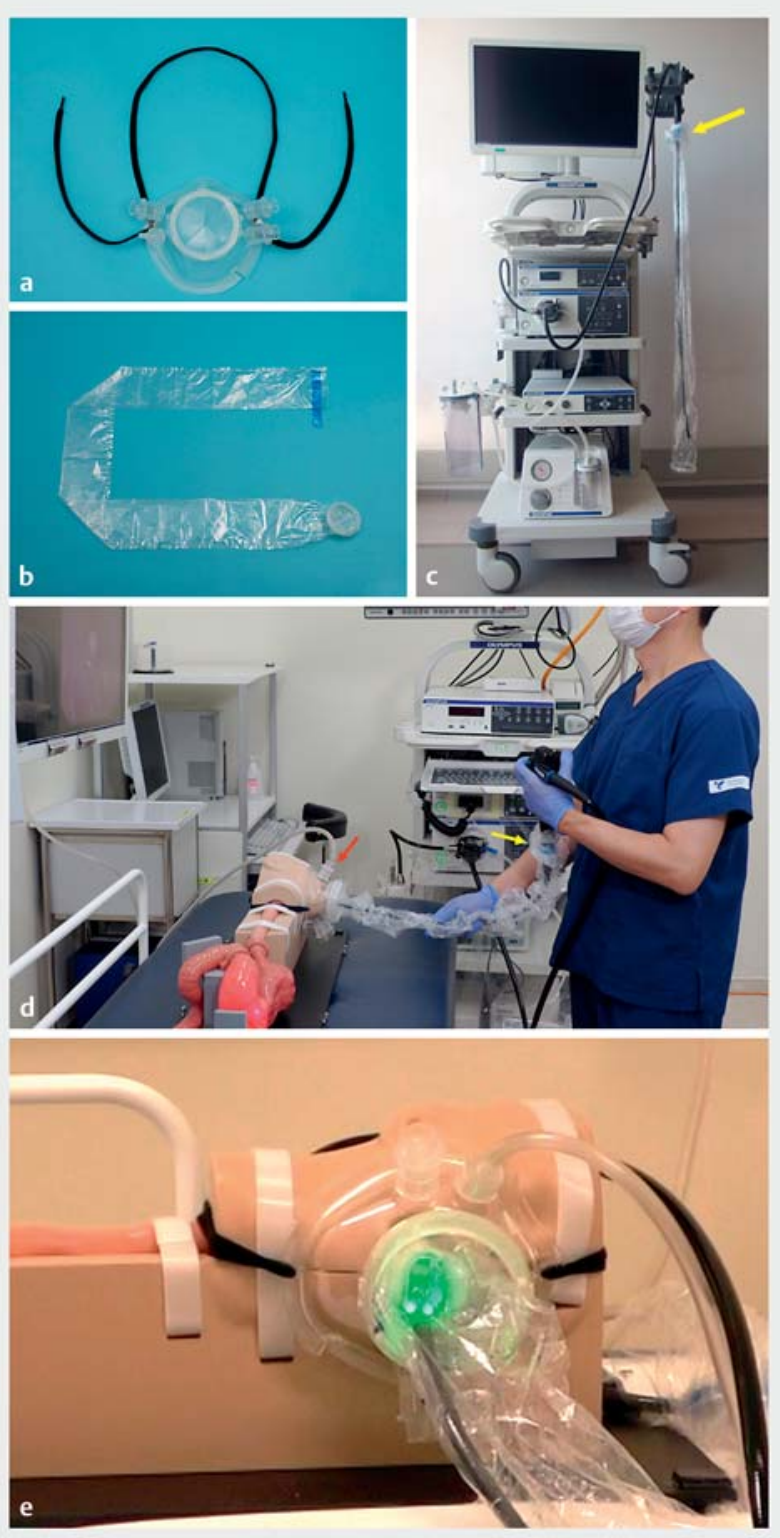

- Fig. 1 STEP ("Shielding Device for the Endoscopic Procedures"). a Mask part, b drape part, and $\mathbf{c}$ endoscope before the examination. The drape was taped to the base of the endoscope (yellow arrow). d Simulated endoscopy using STEP. A suction tube was connected to the mask (red arrow) and aerosols generated from the patient were continuously sucked. e Simulated endoscopy using STEP. The mask part covered the patient's nose and mouth.

ted endoscope remains in the tubular drape while it is transported to the washing room.

\section{Experiment}

In this study, we conducted experiments to investigate the feasibility and utility of STEP in the upper gastrointestinal endoscopy.

For the feasibility study, we used an EVIS Lucera Elite Gastrointestinal Videoscope (GIF-H290Z; Olympus Medical Systems Corp., Tokyo, Japan) and an upper gastrointestinal endoscopy 
training model (LM-103; Koken Co., Ltd., Tokyo, Japan). Three endoscopists performed a total of 18 endoscopic examinations on the training model, alternating between using and not using STEP. The endoscopic examination was performed on the pharynx, the upper middle and lower esophagus, and the stomach. The stomach was observed by the screening method proposed by Yao et al. [20]. Then, the duodenal bulb, descending portion, and Vater's papilla were observed. The procedure time from insertion to removal was compared between the groups using and not using STEP. In addition, three endoscopists who were blinded to the study evaluated endoscopic images of the pharynx/esophagus, the body of the stomach in the retroflex position, the body of the stomach in the straight position, the antrum of the stomach, and the duodenum. The images were evaluated using a visual analog scale (VAS), with scores ranging from 0 ("not observed at all") to 100 ("completely observed").

For the utility study, we created models simulating contact, droplet, and aerosol infection.

In the simulated contact infection model, $20 \mathrm{~mL}$ of indigo carmine pigment was sprayed throughout the stomach of the training model. Then, the three endoscopists, who were wearing white gloves, performed the above-mentioned examination ( $\triangleright$ Fig. 2). The ratio of the area of the pigment attached to the white gloves (the area of the attached pigment / the total area of the glove $\times 100$ ) was measured using image J.

In the simulated droplet infection model ( $\triangleright$ Fig. 3), a sheet of paper was hung on a wall $10 \mathrm{~cm}$ in front of a sprayer installed in the mouth of a polystyrene foam head model, and $0.2 \mathrm{~mL}$ of India ink was sprayed [21]. The paper onto which the India ink was sprayed was then imaged and binarized to divide each pixel into black and white. The experiment was performed three times under the same conditions and the average number of black pixels was compared between trials using and not using STEP.

In the simulated aerosol infection model [22,23], an airway management training model (AirSim Advance Bronchi X; TruCorp., Lurgan, UK) and an electronic cigarette (FLEVO; GIEX, Tokyo, Japan) were used ( $\mathbf{F i g . 4 )}$. The airway management training model was placed inside a plastic case to create a stable environment. The vaporized liquid from the electronic cigarette was sprayed from outside the plastic case as a simulated aerosol for 0.9 seconds and then injected into the respiratory tract through a tube. Using a particle counter (MET ONE HHPC 6+; Beckman Coulter, Brea, California, United States), the total number of particles 0.3 to $10 \mu \mathrm{m}$ in size was measured for 120 seconds. The experiment was performed three times under the same conditions and the average total number of particles was compared between trials using and not using STEP. In the trials using STEP, suction was performed from the suction port of the mask with a pressure of $-40 \mathrm{kPa}$ and a flow rate of $40 \mathrm{~L} / \mathrm{min}$.

\section{Statistical analysis}

Data were analyzed using the unpaired t-test, Chi-squared test, Fisher's test, or Mann-Whitney U-test as appropriate. $\mathrm{P}<0.05$ was considered significant. All statistical analyses were performed using SPSS version 20 (SPSS IBM statistics).

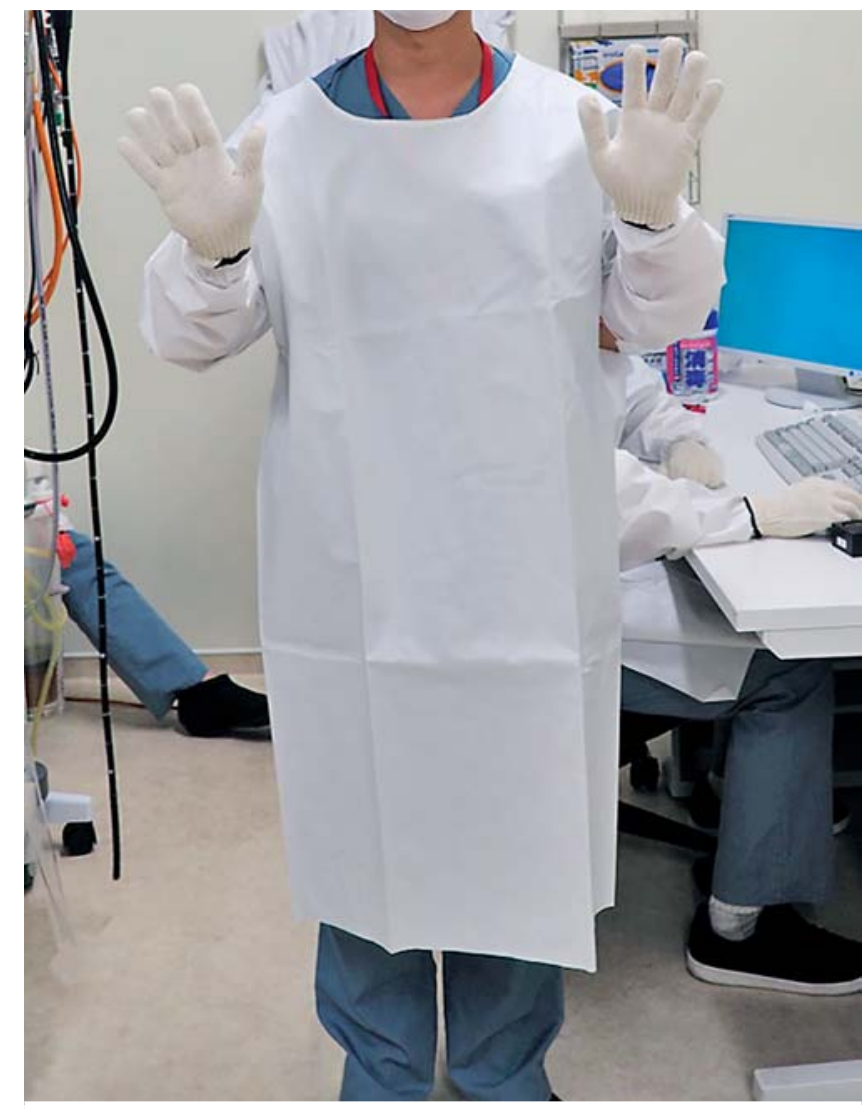

Fig. 2 Simulated contact infection model. After spraying indigo carmine pigment into the training model, the endoscopist put on a white glove and performed an endoscopic screening examination. The proportion of the area of the white glove to which the indigo carmine pigment was attached was measured.

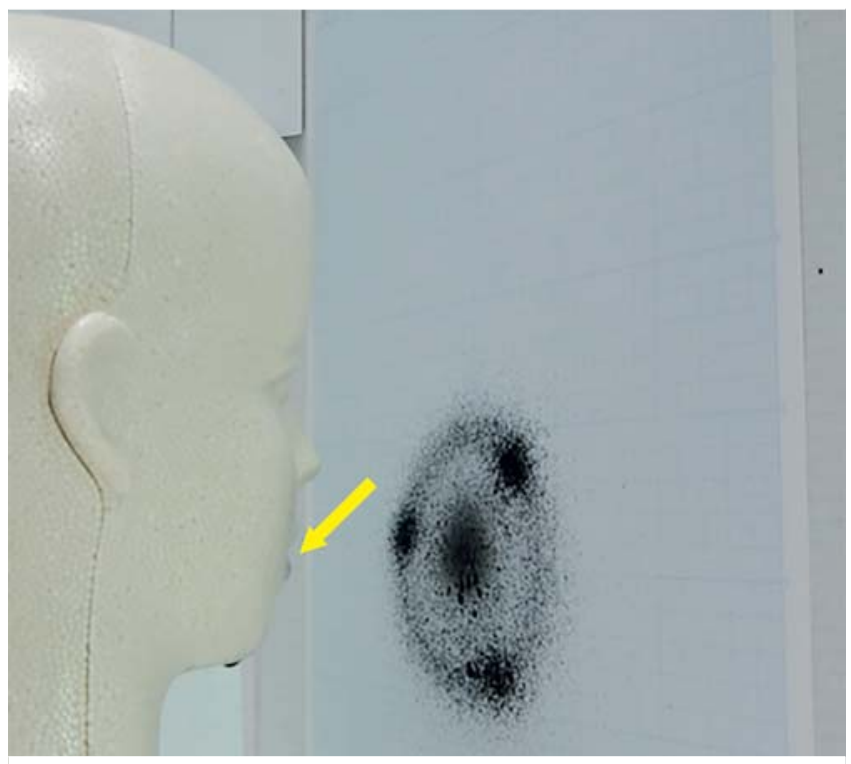

Fig. 3 Simulated droplet infection model. A sprayer was installed in the mouth of the head model (yellow arrow) and ink was sprayed. The paper onto which the ink was sprayed was imaged, and the number of pixels that became black after binarization was measured. 


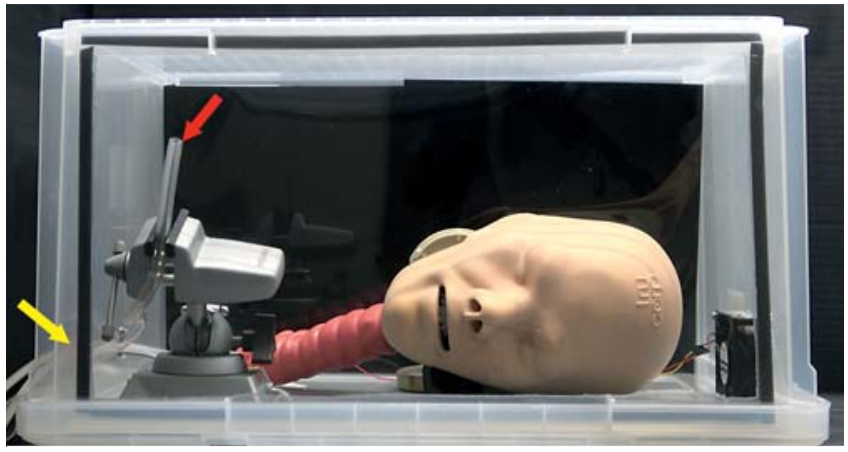

> Fig. 4 Simulated aerosol infection model. The airway management training model was placed inside the plastic case and the vaporized liquid from an e-cigarette was sprayed through a tube from outside the plastic case (yellow arrow). The number of aerosol particles diffused into the plastic case was measured with a particle counter (red arrow).

\section{Results}

A total of 18 endoscopic procedures were performed using STEP without problem. The procedure time was $126.3 \pm 11.6$ seconds with STEP and $122.3 \pm 10.0$ seconds without STEP; no significant difference was observed between the two groups. The VAS scores for the evaluation of endoscopic images taken in the STEP group were as follows: pharynx/esophagus, 91.7 \pm 9.0; body of the stomach in the retroflex position, $91.2 \pm 9.2$; body of the stomach in the straight position, $88.5 \pm 12.3$; antrum of the stomach, $91.0 \pm 9.5$; and duodenum, $91.0 \pm 11.3$. The VAS scores for the non-STEP group were as follows: pharynx/esophagus, $90.9 \pm 9.3$; body of the stomach in the retroflex position, $91.1 \pm 8.8$; body of the stomach in the straight position, 87.7 \pm 13.7 ; antrum of the stomach, $90.9 \pm 9.0$; and duodenum, $91.7 \pm 10.4$. The results were equivalent for the STEP and non-STEP groups ( $\triangleright$ Table 1 ).

In the contact infection model, the proportion of the area of the white gloves onto which the indigo carmine pigment ad- hered was $4.9 \pm 1.4 \%$ in the non-STEP group and $0 \%$ in the STEP group.

In the droplet infection model, the number of pixels that became black after binarization was $351418 \pm 90735$ in the nonSTEP group and 0 in the STEP group.

In the aerosol infection model, the average total number of particles in the plastic case was $346837 \pm 9485$ in the non-STEP group and was significantly reduced to $222 \pm 174$ in the STEP group ( $\triangleright$ Table 2 ).

\section{Discussion}

Endoscopic procedures, especially upper gastrointestinal endoscopy, carry a risk of contact, droplet, and aerosol infection, and thus appropriate infection control measures are required. Current infection control consists of the following three steps: before the procedure, the endoscopy staff disinfects their hands; during the procedure, the endoscopy staff wears PPE; after the procedure, the endoscopy staff cleans the endoscope and endoscopy room and disinfects their hands [24]. However, once contaminants are diffused by coughing or vomiting, it is difficult to completely clean and disinfect objects they come into contact with. Therefore, these three steps are considered to be insufficient as infection control measures. It is necessary to shield the patient in order to prevent as much as possible the spread of contaminants to the surrounding environment. Accordingly, we have developed the STEP shielding device for endoscopic procedures.

Several methods for shielding patients during endoscopy have previously been reported [25-31], including a transparent plastic box and a vinyl sheet, both of which cover the patient. However, these devices need to be cleaned and disinfected if they are reused. Therefore, disposable items are desirable and we developed a solution consisting of a disposable mask and drape. In addition, it was considered that the operability of the endoscope may deteriorate when the patient is covered with a shield during the procedure. We were similarly concerned that STEP might also affect the operability of the endoscope. How-

- Table 1 Results of the feasibility study

\begin{tabular}{|c|c|c|c|}
\hline & $\begin{array}{l}\text { STEP group } \\
(\mathrm{n}=9)\end{array}$ & $\begin{array}{l}\text { Non-STEP group } \\
(n=9)\end{array}$ & $P$ value \\
\hline Procedure time, $s \pm S D$ & $126.3 \pm 11.6$ & $122.3 \pm 10.0$ & N.S. \\
\hline \multicolumn{4}{|l|}{ VAS score } \\
\hline All images & $90.7 \pm 10.1$ & $90.4 \pm 10.0$ & N.S. \\
\hline Pharynx/esophagus & $91.7 \pm 9.0$ & $90.9 \pm 9.3$ & N.S. \\
\hline Body of stomach in retroflex position & $91.2 \pm 9.2$ & $91.1 \pm 8.8$ & N.S. \\
\hline Body of stomach in straight position & $88.5 \pm 12.3$ & $87.7 \pm 13.7$ & N.S. \\
\hline Antrum of stomach & $91.0 \pm 9.5$ & $90.9 \pm 9.0$ & N.S. \\
\hline Duodenum & $91.0 \pm 11.3$ & $91.7 \pm 10.4$ & N.S \\
\hline
\end{tabular}


Table 2 Results of the utility study

\begin{tabular}{|l|l|l|}
\hline & $\begin{array}{l}\text { STEP group } \\
(\mathbf{n = 3})\end{array}$ & $\begin{array}{l}\text { Non-STEP group } \\
\mathbf{( n = 3 )}\end{array}$ \\
\hline $\begin{array}{l}\text { Contact infection model } \\
\text { Proportion of area of the white glove to which the indigo carmine } \\
\text { pigment adhered } \% \pm \text { SD) }\end{array}$ & 0 & $4.9 \pm 1.4$ \\
\hline $\begin{array}{l}\text { Droplet infection model } \\
\text { Number of pixels that became black after binarization (pixels } \pm \text { SD) }\end{array}$ & 0 & $351418 \pm 90735$ \\
\hline $\begin{array}{l}\text { Aerosol infection model } \\
\text { Total number of particles in the plastic case (number } \pm \text { SD) }\end{array}$ & $222 \pm 174$ \\
\hline $\begin{array}{l}\text { Data are shown as the mean } \pm \text { standard deviation. } \\
\text { SD, standard deviation. }\end{array}$ & $346837 \pm 9485$ \\
\hline
\end{tabular}

ever, although the upper gastrointestinal endoscopy took several seconds longer when using STEP, no significant difference was observed between the STEP and non-STEP groups. In addition, the evaluation of endoscopic images produced nearly identical results between the STEP and non-STEP groups. It was considered that the effect of STEP on the operability of the upper endoscopy was minimal.

The primary purpose of shielding is to prevent contact with droplets from the patient's mouth and nose. However, saliva and digestive juices attached to the endoscope can also be a source of infection. STEP's tubular drape is attached to the endoscope and prevents direct contact with saliva and digestive juices. In addition, given that the endoscope can remain inside the tubular drape while being carried to the washing room, it is possible to prevent contamination of the surrounding environment and medical staff after the examination. In other words, STEP can play an important role not only during but also after the endoscopic procedure. In this experiment, there was zero contact with simulated contaminants in the STEP group in both the contact infection and droplet infection models. Endoscopic procedures are also considered to pose a risk of aerosol infection. COVID-19 can be spread by aerosol infection as well as contact and droplet infection. To prevent aerosols from spreading, a suction port was added to the mask. Continuous suction during the procedure significantly reduced the amount of simulated aerosols released into the surrounding environment.

This study has several limitations. First, it is unclear how many droplets and aerosols are generated during actual endoscopic procedures and how infectious they are. In this experiment, an electronic cigarette was used as a simulated aerosol, but there are no data on aerosol generation in clinical practice. In the future, we plan to measure droplets and aerosols the clinical setting and investigate the extent to which they can be contained by STEP. Second, if the patient's oxygen saturation level drops, it is necessary to remove the mask and apply a nasal cannula for supplemental oxygen. At this time, the patient might cough or sneeze and spread droplets and aerosols. Therefore, nasal cannulas should be applied before the start of the procedure when administering sedative agents. In addition, it is considered important to monitor the oxygen saturation level dur- ing an endoscopic procedure performed with STEP. It is expected that the patient might feel some discomfort from wearing the mask during the procedure. Moreover, saliva and vomiting may also collect in the mask, which an increase the patient's discomfort and affect the breathing. Therefore, before introducing STEP into clinical practice, we plan to prospectively evaluate patient discomfort and oxygen saturation level. Third, STEP's tubular cover may affect the operability of the endoscope slightly. In this experiment, no significant difference was observed between screening endoscopy performed with and without STEP, but it is necessary to investigate whether STEP affects operability when used in therapeutic endoscopy. Finally, major issues are cost and the question of whether to use STEP for all examinations and treatments or for only patients at high risk of infection. It is also important to evaluate the time required to prepare and dispose of the STEP when introducing it into clinical practice. Therefore, it will be necessary to evaluate the advantages and disadvantages of STEP, including its cost and time.

\section{Conclusions}

In this study, we developed STEP in collaboration with Olympus Medical Systems Co., Ltd. and an otolaryngologist. When using STEP, the operability of the upper gastrointestinal endoscope and the quality of the obtained endoscopic images were equivalent to the conventional method without STEP. In addition, the use of STEP made it possible to reduce the spread of contaminants in simulated models of contact, droplet, and aerosol infection, suggesting that STEP may be useful for infection control in upper gastrointestinal endoscopy.

\section{Acknowledgements}

The authors would like to thank Dr. Muneyoshi Kimura and Dr. Hideki Araoka for helpful discussions. 


\section{Competing interests}

This research and development was carried out in collaboration with Olympus Medical Systems Corporation and an otolaryngologist (Dr. Matsuwaki). Toranomon Hospital and Olympus Medical Systems Corporation have entered into an advisory agreement regarding the development of the device reported in this study. Toranomon Hospital has received an advisory contact fee from Olympus Medical Co., Ltd. Mr. Ariyoshi, Mr. Morishima, Mr. Kimura and Ms. Iwamoto are employees of Olympus Medical Systems Co., Ltd. Mr. Ezawa is an employee of Olympus Corporation. Dr. Kikuchi received the device from Olympus Medical Systems Co., Ltd.

\section{References}

[1] Chen N, Zhou M, Dong X et al. Epidemiological and clinical characteristics of 99 cases of 2019 novel coronavirus pneumonia in Wuhan, China: a descriptive study. Lancet 2020; 395: 507-513

[2] Anderson EL, Turnham P, Griffin JR et al. Consideration of the aerosol transmission for COVID-19 and public health. Risk Anal 2020; 40: 902-907

[3] Harding $\mathrm{H}$, Broom A, Broom J. Aerosol-generating procedures and infective risk to healthcare workers from SARS-CoV-2: the limits of evidence. J Hosp Infect 2020; 105: 717-725

[4] Tirupathi R, Bharathidasan K, Palabindala V et al. Comprehensive review of mask utility and challenges during the COVID-19 pandemic. Infez Med 2020; 28: 57-63

[5] Cao B, Wang Y, Wen D et al. a trial of lopinavir-ritonavir in adults hospitalized with severe Covid-19. N Engl J Med 2020; 382: 17871799

[6] Grein J, Ohmagari N, Shin D et al. compassionate use of remdesivir for patients with severe Covid-19. N Engl J Med 2020; 382: 2327-2336

[7] Krammer F. SARS-CoV-2 vaccines in development. Nature 2020; 586 : 516-527

[8] Dhama K, Sharun K, Tiwari R et al. COVID-19, an emerging coronavirus infection: advances and prospects in designing and developing vaccines, immunotherapeutics, and therapeutics. Hum Vaccin Immunother 2020; 16: 1232-1238

[9] Mulligan M], Lyke KE, Kitchin N et al. Phase I/II study of COVID-19 RNA vaccine BNT162b1 in adults. Nature 2020; 586: 589-593

[10] Chung YH, Beiss V, Fiering SN et al. COVID-19 Vaccine Frontrunners and Their Nanotechnology Design. ACS Nano 2020; 14: 12522-12537

[11] Amawi H, Abu Deiab GI, A-Aljabali AA et al. COVID-19 pandemic: an overview of epidemiology, pathogenesis, diagnostics and potential vaccines and therapeutics. Ther Deliv 2020; 11: 245-268

[12] Dagotto G, Yu J, Barouch DH. Approaches and challenges in SARSCoV-2 vaccine development. Cell Host Microbe 2020; 28: 364-370

[13] Chiu PWY, $\mathrm{Ng} \mathrm{SC}$, Inoue $\mathrm{H}$ et al. Practice of endoscopy during COVID19 pandemic: position statements of the Asian Pacific Society for Digestive Endoscopy (APSDE-COVID statements). Gut 2020; 69: 991996

[14] Irisawa A, Furuta T, Matsumoto T et al. Gastrointestinal endoscopy in the era of the acute pandemic of COVID-19: Recommendations by Japan Gastroenterological Endoscopy Society. Dig Endosc 2020; 32: 648-650
[15] Gralnek IM, Hassan C, Beilenhoff U et al. ESGE and ESGENA Position Statement on gastrointestinal endoscopy and COVID-19: An update on guidance during the post-lockdown phase and selected results from a membership survey. Endoscopy 2020; 52: 891-898

[16] Sultan S, Lim JK, Altayar O et al. AGA Rapid Recommendations for Gastrointestinal Procedures During the COVID-19 Pandemic. Gastroenterology 2020; 159: 739-758

[17] Chai N, Mei Z, Zhang W et al. Endoscopy works during the pandemic of coronavirus COVID-19: recommendations by the Chinese Society of Digestive Endoscopy. E. United European Gastroenterol J 2020; 8: 798-803

[18] Farneti P, Sorace F, Tasca I. Personal protective equipment for ENT activity during COVID-19 pandemic. Eur Arch Otorhinolaryngol 2020; 277: 2933-2935

[19] Chai N, Tang X, Linghu E et al. The influence of the COVID-19 epidemic on the gastrointestinal endoscopy practice in China: a national survey. Surg Endosc 2020; 11: 1-8

[20] Yao K. The endoscopic diagnosis of early gastric cancer. Ann Gastroenterol 2013; 26: 11-22

[21] Xie X, Li Y, Sun $\mathrm{H}$ et al. Exhaled droplets due to talking and coughing. J R Soc Interface 2009; 6: 703-714

[22] Workman AD, Welling DB, Carter BS et al. Endonasal instrumentation and aerosolization risk in the era of COVID-19: simulation, literature review, and proposed mitigation strategies. Int Forum Allergy Rhinol 2020; 10: 798-805

[23] Sagami R, Nishikiori H, Sato T et al. Aerosols produced by upper gastrointestinal endoscopy: A quantitative evaluation. Am J Gastroenterol 2021; 116: 202-205

[24] Chu DK, Akl EA, Duda S et al. Physical distancing, face masks, and eye protection to prevent person-to-person transmission of SARS-CoV-2 and COVID-19: a systematic review and meta-analysis. Lancet 2020; 395: 1973-1987

[25] De Grazia F, Marconi S, Bardone M et al. Use of 3D printer for face mask production to protect endoscopy unit personnel in contact with high-risk patients during COVID-19 pandemic. Endoscopy 2020; 52: 1146-1147

[26] Zhiqin W, Muhammad Nawawi KN, Raja Ali RA. Application of an antiaerosol box for esophagogastroduodenoscopy during the COVID-19 pandemic: double up the protection. Endoscopy 2020; 52: 704-705

[27] Aponte Martín DM, Corso C, Fuentes C et al. Use of a new face shield for patients of the endoscopy unit to avoid aerosol exchange in the COVID-19 era. VideoGIE 2020; 5: 522-524

[28] Ino Y, Yano T, Yamamoto H. A new simple method of handmade face shield using A4-size OHP sheet, during the COVID-19 pandemic. Dig Endosc 2020; 32: e116-e117

[29] Kobara H, Nishiyama N, Masaki T. Shielding for patients using a singleuse vinyl-box under continuous aerosol suction to minimize SARSCoV-2 transmission during emergency endoscopy. Dig Endosc 2020; 32: e114-e115

[30] Kikuchi D, Suzuki Y, Nomura K et al. New safety measure for the endoscopic procedures during the COVID-19 pandemic: New STEP. VideoGIE 2020; 5: 634-636

[31] Kikuchi D, Suzuki Y, Hoteya S. Shielding method for the endoscopic procedures during the COVID-19 pandemic. Dig Endosc 2020; 32: e160-e161 